\title{
Fractional Order Model of Phytoplankton-toxic Phytoplankton-Zooplankton System
}

\author{
Moustafa El-Shahed $^{1}$, A. M. Ahmed ${ }^{2}$, Ibrahim. M. E. Abdelstar ${ }^{2,3}$ \\ ${ }^{1}$ Department of Mathematics, Faculty of Arts and Sciences Qassim University, P.O. Box 3771, Qassim, Unizah \\ 51911, Saudi Arabia \\ ${ }^{2}$ Department of Mathematics, Faculty of Science, Al-Azhar University, Nasr City, P.O.Box: 11884, Cairo, Egypt \\ ${ }^{3}$ Quantitative Methods Unit, Faculty of Business and Economics Qassim University, P.O.Box: 6633, Qassim, \\ Buridah 51452, Saudi Arabia \\ Email: elshahedm@yahoo.com
}

\begin{abstract}
In this paper, a fractional-order model for phytoplankton-toxic phytoplanktonzooplankton system is presented. This model consists of three components: phytoplankton, toxic phytoplankton, and zooplankton. The equilibrium points are computed and stability of the equilibrium points is analyzed. In addition, fractional Hopf bifurcation conditions for the model are proposed. The generalized Adams-Bashforth-Moulton method is used to solve and simulate the system of fractional differential equations.
\end{abstract}

Keywords: Toxic-phytoplankton, zooplankton, fractional order, stability, numerical method.

\section{Introduction}

Phytoplankton, are the autotrophic (self-feeding) components of the plankton community and a key part of seas, oceans and freshwater basin ecosystems. Most phytoplankton are very small. However, when present in high enough numbers, some varieties may be noticeable as colored patches on the water surface due to the presence of chlorophyll within their cells and accessory pigments (for example phycobiliproteins or xanthophylls) in some species. Phytoplankton also requires inorganic nutrients such as nitrates, phosphates, and sulfur which they convert into proteins, fats, and carbohydrates. In balanced ecological systems, phytoplankton provides food for a wide range of sea creatures including jellyfish, shrimp, whales, and snails. When too many nutrients are available, phytoplankton can produce extremely toxic compounds that have harmful effects on fish, shellfish, mammals, birds, and even people. Furthermore these blooms may grow out of control and form harmful algal blooms (HABs) this statistics according to NOAA's National Centers for Coastal Ocean Science $[1,2]$.

Fractional differential equations have recently been proved to be an effective and valuable modeling tool in various fields of science and engineering. Indeed, we can find numerous applications in polymer rheology, regular variation in thermodynamics, aerodynamics, biophysics, blood flow phenomena, electrodynamics of complex medium, viscoelasticity, electroanalytical chemistry, biology, Bode analysis of feedback amplifiers, capacitor theory, electrical circuits, control theory, fitting of experimental data, etc. see for example $[3,4,5]$, interest in fractional calculus and fractional differential equations has grown dramatically in recent decades [6,7]. The rest of this paper is organized as follows. Section 2 introduces a fractional order model and discusses the blondeness of the solutions of the model. Section 3 discusses the stability of the equilibrium points of the model. Section 4 simulates the dynamics of the system of fractional differential equations using Adams-Bashforth-Moulton algorithm. Section 5 summarizes this paper.

\section{Model Formulation}

Recently, it has been observed that using fractional order differential equations to model real life phenomena in different fields can be very successful $[6,7,8]$. The tritrophic food chain model for 
phytoplankton, toxic phytoplankton and zooplankton can be written as a set of three nonlinear fractional differential equations as follows [9]:

$$
\begin{aligned}
& D_{t}^{\alpha} P=r_{1} P\left(1-\frac{P}{H_{1}}\right)-a P T-c P Z \\
& D_{t}^{\alpha} T=r_{2} T\left(1-\frac{T}{H_{2}}\right)-b P T-T Z \\
& D_{t}^{\alpha} \quad Z=e P Z-T Z-m Z
\end{aligned}
$$

where $D_{t}^{\alpha}$ is the Caputo fractional derivative and all the parameters are assumed to be non-negative.

Lemma. All solutions of system (2.1) which initiate in $\mathbb{R}_{+}^{3}$ are uniformly bounded.

Proof. To get blondeness of solutions of system (2.1), a function W is defined as follows,

$$
W=P+T+\frac{c}{\mathrm{e}} Z
$$

Now, differentiating the above equation with respect to time $t$, it is obtained that,

$$
\begin{aligned}
& \mathrm{D}_{t}^{\alpha} \mathrm{W}=\mathrm{D}_{t}^{\alpha} \mathrm{P}+\mathrm{D}_{t}^{\alpha} \mathrm{T}+\frac{\mathrm{c}}{\mathrm{e}} \mathrm{D}_{t}^{\alpha} \mathrm{Z} \\
& =r_{1} P\left(1-\frac{P}{H_{1}}\right)-(a+b) P T-\mathrm{c} P Z-\left(1+\frac{c}{e}\right) T Z+r_{2} T\left(1-\frac{T}{H_{2}}\right)-\mathrm{c} P Z-\frac{m c}{\mathrm{e}} Z \\
& \leq r_{1} P-\frac{r_{1} P^{2}}{H_{1}}+r_{2} T-\frac{r_{2} T^{2}}{H_{2}}-\frac{m c}{\mathrm{e}} Z \\
& \leq 2 r_{1} P+2 r_{2} T-\left(r_{1} P+r_{2} T+\frac{m c}{\mathrm{e}} Z\right) \\
& \leq 2 k_{1}(P+T)-k_{2}\left(P+T+\frac{c}{\mathrm{e}} Z\right) \\
& =2 k_{1}(P+T)-k_{2} W
\end{aligned}
$$

where $\mathrm{k}_{1}=\max \left\{\mathrm{r}_{1}, \mathrm{r}_{2}\right\}, \mathrm{k}_{2}=\min \left\{\mathrm{r}_{1}, \mathrm{r}_{2}, \mathrm{~m}\right\}$. By Lemma 9 [10], we have

$$
\mathrm{W} \leq \mathrm{W}(0) \mathrm{E}_{\alpha}\left(-\mathrm{k}_{2} \mathrm{t}^{\alpha}\right)+2 \mathrm{k}_{1}\left[\mathrm{P}(0) \mathrm{E}_{\alpha}\left(\mathrm{r}_{1} \mathrm{t}^{\alpha}\right)+\mathrm{T}(0) \mathrm{E}_{\alpha}\left(\mathrm{r}_{2} \mathrm{t}^{\alpha}\right)\right] \mathrm{E}_{\alpha,+1}\left(-\mathrm{k}_{2} \mathrm{t}^{\alpha}\right)=\mathrm{W}_{1}
$$

where $\mathrm{E}_{\alpha}$ is the Mittag-Leffler function. Therefore, all solutions of the model (2.1) with initial conditions in $\Omega=\left\{(\mathrm{P}, \mathrm{T}, \mathrm{Z}) \in \mathrm{W}: 0 \leq \mathrm{W} \leq \mathrm{W}_{1}\right\}$ remain in $\Omega$ for all $\mathrm{t}>0$. Thus, region $\Omega$ is positively invariant with respect to model $(2.1)$.

In the following, we will study the dynamics of system (2.1).

\section{$3 \quad$ Equilibrium Point and Stability}

In the following, we discuss the stability of the commensurate fractional ordered dynamical system:

$$
\mathrm{D}_{\mathrm{t}}^{\alpha} x_{i}=f_{i}\left(x_{1}, x_{2}, x_{3}\right), \quad \alpha \in(0,1) \quad 1 \leq i \leq 3
$$

Let $\mathrm{E}=\left(x_{1}^{*}, x_{2}^{*}, x_{3}^{*}\right)$ be an equilibrium point of system (3.1) and $x_{i}=x_{i}^{*}+\eta_{i}$, where $\eta_{\mathrm{i}}$ is a small disturbance from a fixed point. Then

$$
\begin{aligned}
& \mathrm{D}_{\mathrm{t}}^{\alpha} \eta_{i}=\mathrm{D}_{\mathrm{t}}^{\alpha} x_{i} \\
& =f_{i}\left(x_{1}^{*}+\eta_{1}, x_{2}^{*}+\eta_{2}, x_{3}^{*}+\eta_{3}\right) \\
& \approx \eta_{1} \frac{\partial f_{i}(E)}{\partial x_{1}}+\eta_{2} \frac{\partial f_{i}(E)}{\partial x_{2}}+\eta_{3} \frac{\partial f_{i}(E)}{\partial x_{3}}
\end{aligned}
$$


System (3.2) can be written as:

$$
\mathrm{D}_{\mathrm{t}}^{\alpha}=\mathrm{Jn}
$$

where $\eta=\left(\eta_{1}, \eta_{2}, \eta_{3}\right)^{T}$ and $J$ is the Jacobian matrix evaluated at the equilibrium points. Using Matignon's results [11], it follows that the linear autonomous system (3.3) is asymptotically stable if $|\arg (\lambda)|>\frac{\alpha \Pi}{2}$ is satisfied for all eigenvalues of matrix $\mathrm{J}$ at the equilibrium point $\mathrm{E}=\left(x_{1}^{*}, x_{2}^{*}, x_{3}^{*}\right)$. If $\Phi(x)=x^{3}+a_{1} x^{2}+a_{2} x+a_{3}$, let $\mathrm{D}(\Phi)$ denote the discriminant of a polynomial $\Phi$, then

$$
D(\Phi)=-\left|\begin{array}{ccccc}
1 & a_{1} & a_{2} & a_{3} & 0 \\
0 & 1 & a_{1} & a_{2} & a_{3} \\
3 & 2 a_{1} & a_{2} & 0 & 0 \\
0 & 3 & 2 a_{1} & a_{2} & 0 \\
0 & 0 & 3 & 2 a_{1} & a_{2}
\end{array}\right|=18 a_{1} a_{2} a_{3}+\left(a_{1} a_{2}\right)^{2}-4 a_{3} a_{1}^{3}-4 a_{2}^{3}-27 a_{3}^{2}
$$

Following $[3,11,12,13]$, we have the proposition.

Proposition 3.1. One assumes that $\mathrm{E}$ exists in $\mathrm{R}_{+}^{3}$.

1. If the discriminant of $\Phi(\mathrm{x}), \mathrm{D}(\Phi)$ is positive and Routh-Hurwitz is satisfied, that is, $D(\Phi)>0, a_{1}>0, a_{3}>0, a_{1} a_{2}>a_{3}$, then $\mathrm{E}$ is locally asymptotically stable.

2. If $\mathrm{D}(\Phi)<0, a_{1}>0, a_{2}>0, a_{1} a_{2}=a_{3}, \alpha \in[0,1)$ then $\mathrm{E}$ is locally asymptotically stable.

3. If $\mathrm{D}(\Phi)<0, a_{1}<0, a_{2}\langle 0, \alpha\rangle \frac{2}{3}$, then $\mathrm{E}$ is unstable.

4. The necessary condition for the equilibrium point $\mathrm{E}$ to be locally asymptotically stable is $a_{3}>0$.

To evaluate the equilibrium points, let

$$
D_{t}^{\alpha} P=0, D_{t}^{\alpha} T=0, D_{t}^{\alpha} Z=0
$$

Then

1. The first trivial equilibrium point is $E_{0}=(0,0,0)$. The point $E_{0}$ always exists.

2. The second equilibrium point is $E_{1}=\left(H_{1}, 0,0\right)$. The point $E_{1}$ always exists.

3. The third equilibrium point is $E_{2}=\left(0, H_{2}, 0\right)$. The point $E_{2}$ always exists.

4. The fourth equilibrium point is $E_{3}=\left(P_{3}, T_{3}, 0\right)$, where

$$
\begin{gathered}
T_{3}=\frac{r_{1} H_{2}\left(r_{2}-b H_{1}\right)}{r_{1} r_{2}-a b H_{1} H_{2}}=\frac{r_{1}\left(R_{0}-1\right)}{a\left(R_{0} R_{1}-1\right)} \\
P_{3}=\frac{r_{2}}{b}\left(1-\frac{T_{3}}{H_{2}}\right)=\frac{r_{2}}{b}\left(1-\frac{r_{1}\left(R_{0}-1\right)}{a H_{2}\left(R_{0} R_{1}-1\right)}\right)
\end{gathered}
$$

and $R_{1}=\frac{r_{1}}{a H_{2}}$ is the reproduction numbers while $R_{1}=\frac{r_{2}}{b H_{1}}$ is the basic reproduction number, see [9]. The point $E_{3}$ exists if $R_{1}>1$ and $R_{0}>1$.

5. The fifth equilibrium point is $E_{4}=\left(P_{4}, 0, Z_{4}\right)$, where $P_{4}=\frac{m}{c}, Z_{4}=\frac{r_{1}}{c}\left(1-R_{0}^{*}\right), R_{0}^{*}=\frac{m}{e H_{1}}$. The point $E_{4}$ exists if $R_{0}^{*}<1$ and 


$$
\begin{gathered}
\frac{b P_{5}}{1-\frac{T_{5}}{H_{2}}}<r_{2}<\min \left\{\frac{a m+r_{1}}{\left.c\left(1-\frac{m}{H_{2}}\right), \frac{H_{2}}{e c}\left(\frac{r_{1}}{H_{1}}-b e+a e\right), \frac{1}{e c}\left(r_{1} e+b c m-\frac{r_{1} m}{H_{1}}\right)\right\} \text { or }}\right. \\
\quad \min \left\{\frac{1}{e c}\left(r_{1} e+b c m-\frac{r_{1} m}{H_{1}}\right), \frac{b P_{5}}{1-\frac{T_{5}}{H_{2}}}, \frac{a m+r_{1}}{c\left(1-\frac{c m}{H_{2}}\right)}, \frac{H_{2}}{e c}\left(\frac{r_{1}}{H_{1}}-b e+a e\right)\right\}<r_{2}
\end{gathered}
$$

6. The sixth interior equilibrium point is $E_{5}=\left(P_{5}, T_{5}, Z_{5}\right)$, where

$$
\begin{gathered}
P_{5}=\frac{a m+r_{1}-r_{2} c-\frac{r_{2} c m}{H_{2}}}{\frac{r_{1}}{H_{1}}-\frac{r_{2} c e}{H_{2}}-b c+a e} \\
T_{5}=e P_{5}-m \\
Z_{5}=r_{2}\left(1-\frac{T_{5}}{H_{2}}\right)-b P_{5}
\end{gathered}
$$

Following Javidi[9], the equilibrium point $\mathrm{E}_{0}$ is a saddle point, $\mathrm{E}_{1}$ is locally asymptotically stable if $\mathrm{R}_{0}<1$ and $\mathrm{R}_{0}^{*}>1$. The equilibrium point $\mathrm{E}_{2}$ is locally asymptotically stable if $\mathrm{R}_{1}<1$ and $\mathrm{E}_{3}$ is locally asymptotically stable if $\mathrm{T}_{3}>\frac{\mathrm{er}_{2} \mathrm{H}_{2}-\mathrm{mbH}_{2}}{\mathrm{bH}_{2}+\mathrm{er}_{2}}$.

Javidi[9] introduced the following theorem:

Theorem 3.1. The equilibrium $E_{4}$ is locally asymptotically stable if

$$
\begin{gathered}
\frac{-2 m}{r_{1}}+2 \sqrt{\left(\frac{m}{r_{1}}\right)^{2}+\frac{m}{r_{1}}}<\mathrm{R}_{0}^{*}<1 \\
\mathrm{R}_{0}^{*}<1-\frac{c}{r_{1}}\left(\mathrm{r}_{2}-\frac{b m}{e}\right)
\end{gathered}
$$

If one takes the same parameters as in [9] which are used in figure 1 [9] except $e, H_{1}$ (because the condition of theorem 8 must be satisfied), where we take $e=1.75, H_{1}=0.8$, then we get

$$
\mathrm{R}_{0}^{*}=0.5714, \frac{-2 m}{r_{1}}+2 \sqrt{\left(\frac{m}{r_{1}}\right)^{2}+\frac{m}{r_{1}}}=0.7136,1-\frac{c}{r_{1}}\left(\mathrm{r}_{2}-\frac{b m}{e}\right)=1.0064
$$

i.e.

$$
\mathrm{R}_{0}^{*}<\min \left\{\frac{-2 m}{r_{1}}+2 \sqrt{\left(\frac{m}{r_{1}}\right)^{2}+\frac{m}{r_{1}}}, 1-\frac{c}{r_{1}}\left(\mathrm{r}_{2}-\frac{b m}{e}\right)\right\}
$$

and

$$
\Pi=-1.410612245<0
$$

in this case, the eigenvalues of $E_{4}$ are

$$
\lambda_{41}=-0.58, \lambda_{42}, \lambda_{43}=-0.5142857143 \pm 0.5938459912 i
$$

and

$$
\alpha^{*}=-0.54555821
$$


which contradicts with the fact that the critical value $\alpha^{*}$ must be in the interval $(0,1)$.

The Jacobian matrix $J\left(E_{4}\right)$ evaluated at an interior equilibrium point $E_{4}$ is given as: $\left(P_{4}, 0, Z_{4}\right)$

$$
J\left(E_{4}\right)=\left(\begin{array}{ccc}
-\frac{r_{1}}{H_{1}} P_{4} & -a P_{4} & -c P_{4} \\
0 & \mathrm{r}_{2}-b P_{4}-Z_{4} & 0 \\
e Z_{4} & -Z_{4} & 0
\end{array}\right)
$$
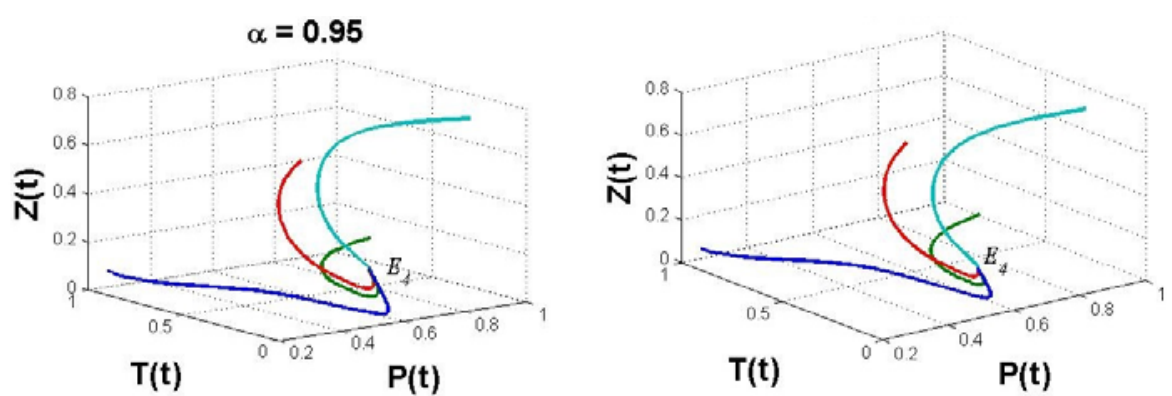

$\alpha=0.75$
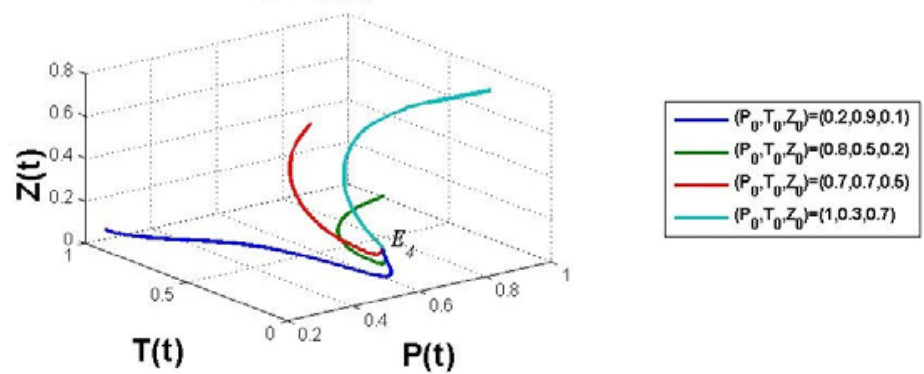

$T(t)$

$\mathrm{P}(\mathrm{t})$

Figure 1. For various initial conditions and $\alpha$, the trajectory of system converges to the equilibrium point E1.

Theorem 3.2. The equilibrium $E_{4}$ is locally asymptotically stable for all $\alpha \in(0,1)$ if the following condition holds

$$
\mathrm{R}_{0}^{*}<\min \left\{\frac{-2 m}{r_{1}}+2 \sqrt{\left(\frac{m}{r_{1}}\right)^{2}+\frac{m}{r_{1}}}, 1-\frac{c}{r_{1}}\left(\mathrm{r}_{2}-\frac{b m}{e}\right)\right\}
$$

Proof. The characteristic equation corresponding to the equilibrium $E_{4}$ is given by

$$
\left[\lambda-\left(\mathrm{r}_{2}-b P_{4}-Z_{4}\right)\right]\left[\lambda^{2}+\frac{r_{1}}{H_{1}} P_{4} \lambda+c e P_{4} Z_{4}\right]=0
$$

If $R_{0}^{*}<1-\frac{c}{r_{1}}\left(r_{2}-\frac{b m}{e}\right)$ then the first eigen value $\lambda_{41}=r_{2}-b P_{4}-Z_{4}=r_{2}-\frac{b m}{e}-\frac{r_{1}}{c}\left(1-R_{0}^{*}\right)<0$.

If $R_{0}^{*}>\frac{-2 m}{r_{1}}+2 \sqrt{\left(\frac{m}{r_{1}}\right)^{2}+\frac{m}{r_{1}}}$ the eigenvalues corresponding to the equilibrium $E_{4}$ are

$$
\lambda_{42}, \lambda_{43}=\frac{1}{2}\left(-\frac{r_{1}}{H_{1}} P_{4} \pm i \sqrt{-\Pi}\right)
$$


Then all the eigenvalues corresponding to the equilibrium $E_{4}$ are negative real number or complex number with negative real part then the condition $\left|\arg \left(\lambda_{4 i}\right)\right|>\frac{\alpha \Pi}{2} ; i=1,2,3$ satisfied for all $\lambda_{4 i}, i=1,2,3$. This completes the proof.

The Jacobian matrix $J\left(E_{5}\right)$ evaluated at an interior equilibrium point $E_{5}$ is given as:

$$
J\left(E_{4}\right)=\left(\begin{array}{ccc}
-\frac{r_{1}}{H_{1}} P_{5} & -a P_{5} & -c P_{5} \\
-b T_{5} & -\frac{r_{2}}{H_{2}} T_{5} & -T_{5} \\
e Z_{5} & -Z_{5} & 0
\end{array}\right)
$$

The characteristic equation of $J\left(E_{5}\right)$ is

$$
\lambda^{3}+A \lambda^{2}+B \lambda+C=0
$$

where

$$
\begin{aligned}
A & =\frac{r_{1}}{H_{1}} P_{5}+\frac{r_{2}}{H_{2}} T \\
B & =\left(\frac{r_{1} r_{2}}{H_{1} H_{2}}-a b\right) T_{5} P_{5}-T_{5} Z_{5}+c e Z_{5} P_{5} \\
C & =P_{5} T_{5} Z_{5}\left(b c-a e-\frac{r_{1}}{H_{1}}+\frac{c \mathrm{c} r_{2}}{H_{2}}\right)
\end{aligned}
$$

The characteristic equation (3.4) has the following roots

$$
\lambda_{51}=\sigma_{2}-\sigma_{1}+\frac{\sigma_{3}}{\sigma_{2}}
$$

and

$$
\lambda_{52}, \lambda_{53}=-\frac{1}{2}\left[2 \sigma_{1}+\sigma_{2}+\frac{\sigma_{3}}{\sigma_{2}} \pm \sqrt{3}\left(\sigma_{2}-\frac{\sigma_{3}}{\sigma_{2}}\right) i\right]
$$

where

$$
\begin{aligned}
& \sigma_{1}=\frac{A}{3} \\
& \sigma_{2}=\sqrt[3]{\sqrt{\left(\frac{A^{3}}{27}+\frac{A B}{6}-\frac{C}{2}\right)^{2}-\sigma_{3}^{3}}-\frac{A^{3}}{27}+\frac{A B}{6}-\frac{C}{2}} \\
& \sigma_{3}=\frac{A^{2}}{9}-\frac{B}{3}
\end{aligned}
$$

Theorem 3.3. With respect to system (2.1), if $\sigma_{2}-\sigma_{1}+\frac{\sigma_{3}}{\sigma_{2}}<0$, the following statements can be obtained.

(a)The equilibrium $E_{5}$ is locally asymptotically stable, for any $\alpha \in(0,1)$. 
(b)If $0<\sqrt{3}\left(\sigma_{2}-\frac{\sigma_{3}}{\sigma_{2}}\right)<2 \sigma_{1}+\sigma_{2}+\frac{\sigma_{3}}{\sigma_{2}}$, the equilibrium $E_{5}$ is locally asymptotically stable if and only if $\alpha \in\left(0, \alpha^{*}\right)$, where $\alpha^{*}=\frac{2}{\pi}\left|\tan ^{-1}\left(\frac{\sqrt{3}\left(\sigma_{2}-\frac{\sigma_{3}}{\sigma_{2}}\right)}{2 \sigma_{1}+\sigma_{2}+\frac{\sigma_{3}}{\sigma_{2}}}\right)\right|$

(c)If $\sqrt{3}\left(\sigma_{2}-\frac{\sigma_{3}}{\sigma_{2}}\right) \geq 2 \sigma_{1}+\sigma_{2}+\frac{\sigma_{3}}{\sigma_{2}}$, the equilibrium $E_{5}$ is unstable for any $\alpha \in(0,1)$.

Proof. The conclusions (a) and (c) are obvious. For the statement (b), due to $0<\sqrt{3}\left(\sigma_{2}-\frac{\sigma_{3}}{\sigma_{2}}\right)<2 \sigma_{1}+\sigma_{2}+\frac{\sigma_{3}}{\sigma_{2}}$, the equation (3.5) has two complex roots $\lambda_{52}, \lambda_{53}$, and their real part is $\frac{\sqrt{3}}{2}\left(\sigma_{2}-\frac{\sigma_{3}}{\sigma_{2}}\right)>0$. Then $\left|\arg \left(\lambda_{5 i}\right)\right|=\tan ^{-1}\left(\frac{\sqrt{3}\left(\sigma_{2}-\frac{\sigma_{3}}{\sigma_{2}}\right)}{2 \sigma_{1}+\sigma_{2}+\frac{\sigma_{3}}{\sigma_{2}}}\right), j=2,3$. Besides, according to the condition $\tan ^{-1}\left(\frac{\sqrt{3}\left(\sigma_{2}-\frac{\sigma_{3}}{\sigma_{2}}\right)}{2 \sigma_{1}+\sigma_{2}+\frac{\sigma_{3}}{\sigma_{2}}}\right)=\frac{\alpha^{*} \pi}{2}, \alpha \in\left(0, \alpha^{*}\right)$ if and only if $\left|\arg \left(\lambda_{5 i}\right)\right|>\frac{\alpha \pi}{2}, j=2,3$, it is concluded that Theorem 3.3 is true.

It can be concluded from the statements of theorem 3.4 that the positive equilibrium is locally asymptotically stable if and only if $\alpha \in\left(0, \alpha^{*}\right)$. At $\alpha=\alpha^{*}$ a Hopf bifurcation is expected to occur. As increases above the critical value $\alpha^{*}$, the positive equilibrium is unstable, and a limit cycle is expected to appear in the proximity of $\boldsymbol{E}_{2}$ due to the Hopf bifurcation phenomenon.

The analysis of periodic solutions in fractional dynamical systems is a new research topic that is generating great interest. Thus far, research indicates that exact periodic solutions in time invariant fractional systems are non-existent. In terms of its application, the limit cycle detected in numerical simulations of a simple fractional neural network cannot represent an exact periodic solution of the system. Other research studies have also provided numerical evidence of limit cycles.

\section{$4 \quad$ Numerical Method and Simulation}

Because most fractional-order differential equations lack exact analytic solutions, approximation and numerical techniques must be used. There exist several different analytical and numerical methods for solving fractional-order differential equations. For numerical solutions of system (2.1), one can use the generalized Adams-Bashforth-Moulton method. To give the approximate solution by means of this algorithm, consider the following nonlinear fractional-order differential equation [10, 14-23]:

$$
\begin{aligned}
& D_{t}^{\alpha} y(t)=f(t, y(t)), \quad 0 \leq t \leq T \\
& y^{(k)}(0)=y_{0}^{k}, k=0,1,2, \ldots \ldots, m-1, m-1<\alpha \leq m
\end{aligned}
$$

This equation is equivalent to the Volterra integral equation:

$$
y(t)=\sum_{k=0}^{m-1} y_{0}^{(k)} \frac{t^{k}}{k !}+\frac{1}{\Gamma(\alpha)} \int_{0}^{t}(t-s)^{\alpha-1} f(s, y(s)) d s
$$


Diethelm et al. used the predictor-corrector scheme [14, 15], based on the Adams-Bashforth-Moulton algorithm, to integrate Eq. (4.1). By applying this scheme to the fractional-order model for a tritrophic model consisting of phytoplankton, toxic phytoplankton and zooplankton, and setting $\mathrm{h}=\frac{\mathrm{T}}{\mathrm{N}}, \mathrm{t}_{\mathrm{n}}=\mathrm{nh}$, $\mathrm{n}=0,1,2, \ldots, \mathrm{N} \in \mathrm{Z}^{+}$, one can discretize Eq. (4.1) as follows:

$$
\begin{aligned}
& P_{n+1}= P_{0}+\frac{h^{\alpha}}{\Gamma(\alpha+2)}\left[r_{1} P_{n+1}^{p}\left(1-\frac{P_{n+1}^{p}}{H_{1}}\right)-a P_{n+1}^{p} T_{n+1}^{p}-c P_{n+1}^{p} Z_{n+1}^{p}\right] \\
&+ \frac{h^{\alpha}}{\Gamma(\alpha+2)} \sum_{j=1}^{n} a_{j, n+1}\left[r_{1} P_{j}\left(1-\frac{P_{j}}{H_{1}}\right)-a P_{j} T_{j}-c P_{j} Z_{j}\right] \\
& T_{n+1}= T_{0}+\frac{h^{\alpha}}{\Gamma(\alpha+2)}\left[r_{2} T_{n+1}^{p}\left(1-\frac{T_{n+1}^{p}}{H_{2}}\right)-b P T_{n+1}^{p}-T_{n+1}^{p} Z_{n+1}^{p}\right] \\
&+\frac{h^{\alpha}}{\Gamma(\alpha+2)} \sum_{j=1}^{n} a_{j, n+1}\left[r_{2} T_{j}\left(1-\frac{T_{j}}{H_{2}}\right)-b P_{j} T_{j}-T_{j} Z_{j}\right] \\
& Z_{n+1}=Z_{0}+\frac{h^{\alpha}}{\Gamma(\alpha+2)}\left[e P_{n+1}^{p} Z_{n+1}^{p}-T_{n+1}^{p} Z_{n+1}^{p}-m Z_{n+1}^{p}\right]+\frac{h^{\alpha}}{\Gamma(\alpha+2)} \sum_{j=1}^{n} a_{j, n+1}\left[e P_{j} Z_{j}-T_{j} Z_{j}-m Z_{j}\right]
\end{aligned}
$$

where

$$
\begin{gathered}
P_{n+1}^{p}=P_{0}+\frac{1}{\Gamma(\alpha)} \sum_{j=0}^{n} b_{j, n+1}\left[r_{1} P_{j}\left(1-\frac{P_{j}}{H_{1}}\right)-a P_{j} T_{j}-c P_{j} Z_{j}\right] \\
T_{n+1}^{p}=T_{0}+\frac{1}{\Gamma(\alpha)} \sum_{j=0}^{n} b_{j, n+1}\left[r_{2} T_{j}\left(1-\frac{T_{j}}{H_{2}}\right)-b P_{j} T_{j}-T_{j} Z_{j}\right] \\
Z_{n+1}^{p}=Z_{0}+\frac{1}{\Gamma(\alpha)} \sum_{j=0}^{n} b_{j, n+1}\left[e P_{j} Z_{j}-T_{j} Z_{j}-m Z_{j}\right] \\
a_{j, n+1}=\left\{\begin{array}{l}
(n-j-2)^{\alpha+1}+(n-j)^{\alpha+1}-2(n-j+1)^{\alpha+1} \quad 1 \leq j \leq n \\
1 \quad j=0 \\
n_{j, n+1}^{\alpha-1}=\frac{h^{\alpha}}{\alpha}\left[(n-j+1)^{\alpha}-(n-j)^{\alpha}\right], 0 \leq j \leq n
\end{array}\right] \quad j=0
\end{gathered}
$$

In this section, the dynamical behavior of the proposed model (2.1) has been discussed numerically using MATLAB. For the parameters set, $r_{1}=0.08, r_{2}=0.22, a=0.1, b=0.8$, $c=1.35, e=0.63, m=0.8, H_{2}=0.13$ and $\mathrm{H}_{1}=1$. Now, for this data, it is seen that $\mathrm{R}_{0}=0.2750<1$ and $R_{0}^{*}=1.2698>1$. So according to stability conditions the equilibrium point $E_{1}=(1,0,0)$ is locally asymptotically stable. In Figure 2, we considered four different initial values of the phytoplankton, toxic phytoplankton and zooplankton. All trajectories starting from different initial values approach to the equilibrium point $\mathrm{E}_{1}=(1,0,0)$. 

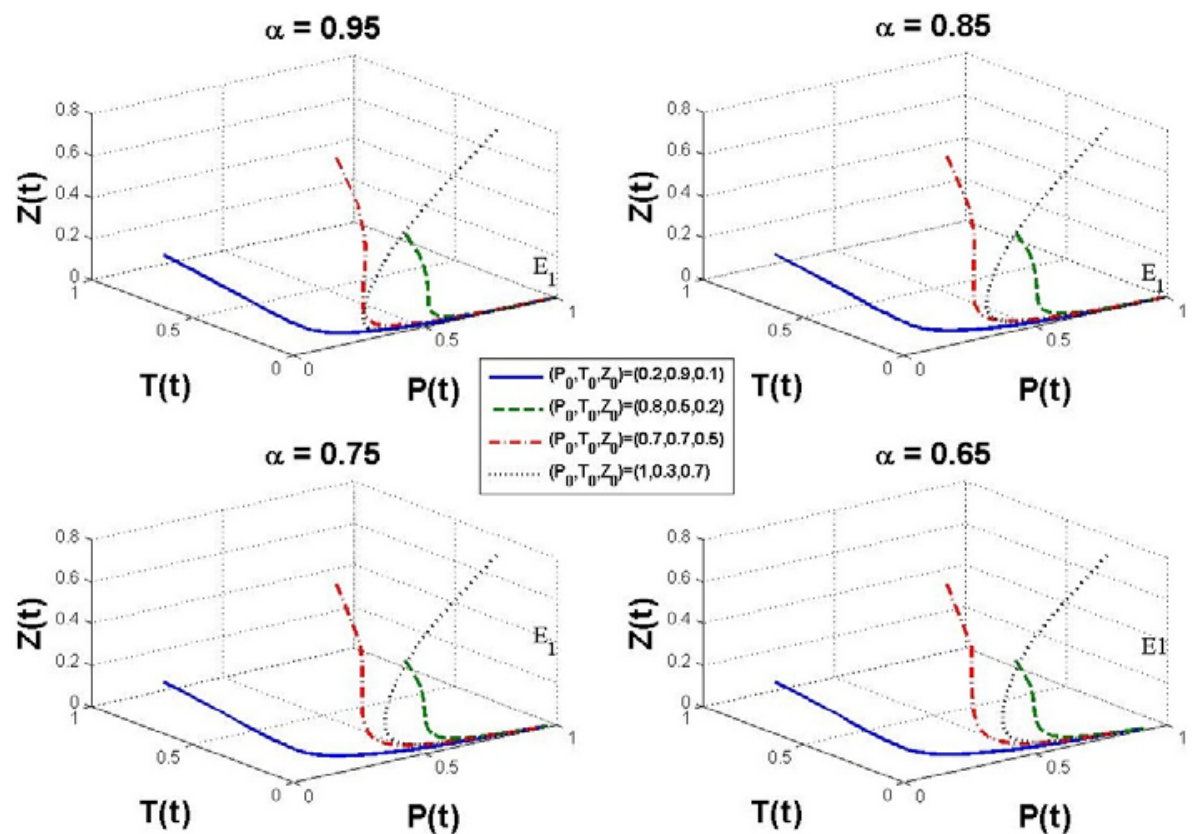

Figure 2. For various initial conditions and a, the trajectory of system converges to the equilibrium point $\mathrm{E}_{1}$.

All the details related to initial values are shown in the legend. Again for the set of parametric values $r_{1}=0.01, r_{2}=0.22, a=0.1, b=0.8, c=1.35, e=0.63, m=0.8, H_{2}=0.5$ and $\mathrm{H}_{1}=0.1$, it is seen that $\mathrm{R}_{1}=0.2<1$. From fig. 3 , it is seen that the equilibrium point $\mathrm{E}_{2}=\left(0, \mathrm{H}_{2}, 0\right)$ is locally asymptotically stable for $\alpha=0.85,0.75$ and 0.65 . In fig. 4 the trajectory of system (2.1) is shown when $r_{1}=0.5, r_{2}=0.92, a=0.2, b=0.5, c=1.35, e=0.63, m=0.8, H_{2}=0.9$ and $\mathrm{H}_{1}=0.6 \quad$. In this case $\mathrm{R}_{0}=3.0667>1$ and $\mathrm{R}_{0}^{*}=2.7778>1$ and the equilibrium point $\mathrm{E}_{3}=(0.4351,0.6872,0)$ is locally asymptotically stable. Again, the following set of parameters is considered: such as $r_{1}=1.8, r_{2}=0.22, a=0.2, b=0.5, c=1.35, e=1.63, m=0.8, H_{2}=0.9$ and $\mathrm{H}_{1}=0.6$. For these parametric values, it is seen that

$$
\mathrm{R}_{0}^{*}=0.818\left\langle 1, \mathrm{R}_{0}^{*}-\left(2 \sqrt{\left(\frac{\mathrm{m}}{\mathrm{r}_{1}}\right)^{2}+\frac{\mathrm{m}}{\mathrm{r}_{1}}}-\frac{2 \mathrm{~m}}{\mathrm{r}_{1}}\right)=0.1044\right\rangle 0
$$

and

$$
\mathrm{R}_{0}^{*}-\left[1-\frac{\mathrm{c}}{\mathrm{r}_{1}}\left(\mathrm{r}_{2}-\frac{\mathrm{bm}}{\mathrm{e}}\right)\right]=-0.2681<0
$$

According to the theorem 3.3 , the equilibrium point $\mathrm{E}_{4}=(0.4908,0,0.2427)$, is locally asymptotically stable. Now, the following parametric values have been considered to discuss the dynamical behavior of the fractional order model taking $\alpha$ as a bifurcation parameter. $r_{1}=0.08, r_{2}=0.3, a=0.1, b=0.4, c=0.79, e=4.8, m=0.8, H_{2}=0.46$ and $H_{1}=1 \quad$. In this case $\alpha^{*}=0.9794388315$. From fig.5.1, it is seen that the trajectory of system (2.1) converges to the equilibrium point $E_{5}=(0.2195,0.2534,0.047)$ for $\alpha=0.95$. Fig.5.2 shows that the trajectory of system (2.1) converges to an asymptotically stable limit cycle $E_{5}=(0.2195,0.2534,0.047)$ for $\alpha=0.99$. These two different behavior are shown in Figures 6-1 and 6-2 for phytoplankton, toxic phytoplankton and zooplankton respectively. From Fig.7, it is clear that the approximate solutions depend continuously on the fractional derivative $\alpha$. 

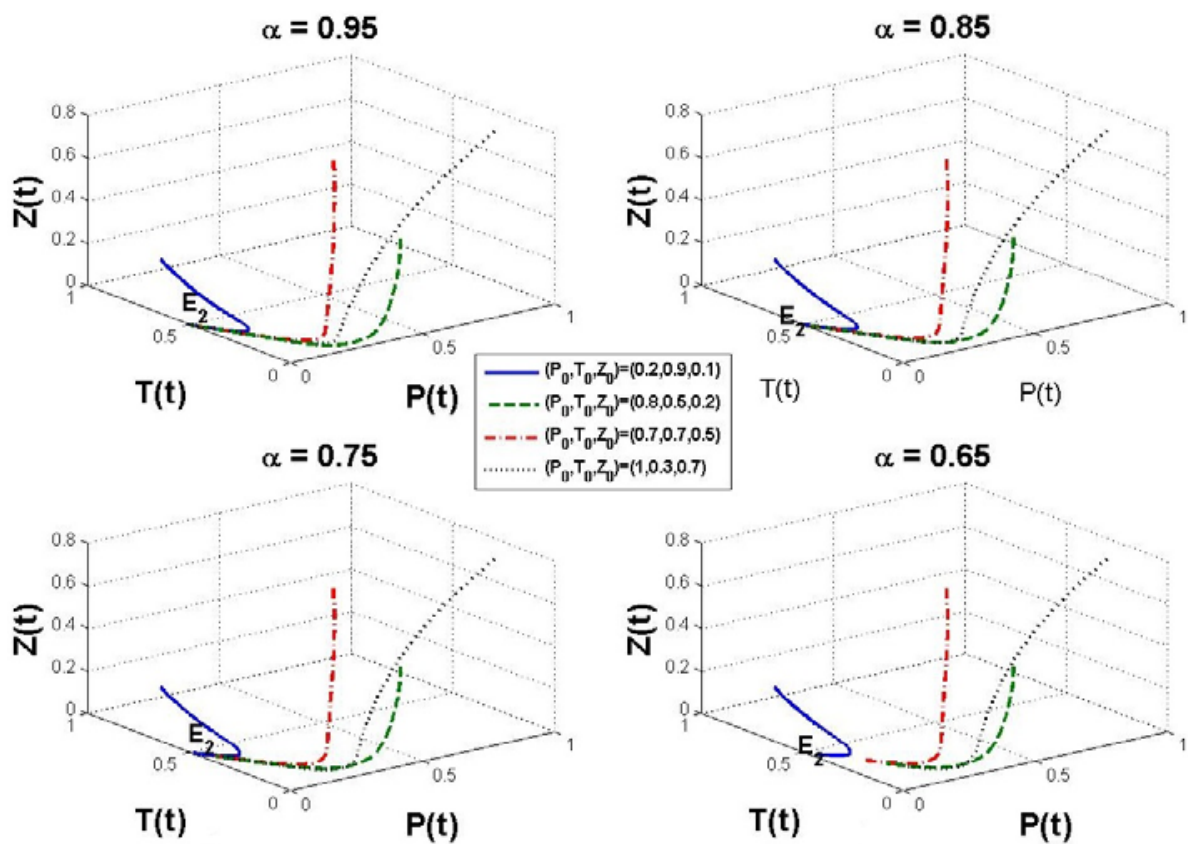

Figure 3. For various initial condition and $\alpha$, the trajectory of system converges to the equilibrium point $E_{2}$.
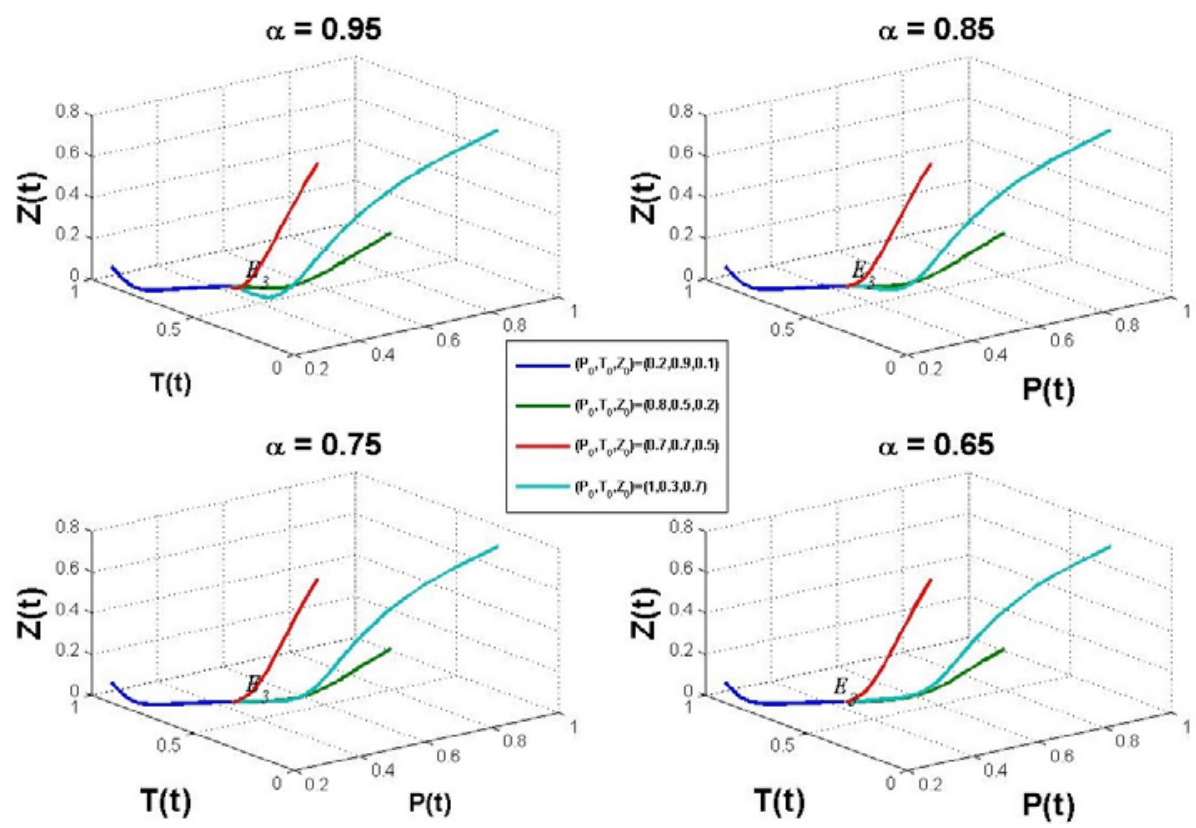

Figure 4. For various initial conditions and $\alpha$, the trajectory of system converges to the equilibrium point $E_{3}$.

\section{Conclusion}

In [9], the authors introduced a fractional order phytoplankton toxic-phytoplankton zooplankton system and provide some numerical simulations. In [9, 24-27], the authors used a particular numerical scheme according to Atanackovic and Stankovich in $[28,29]$. This method is not proved to be convergent and 
deserves more study as already pointed out in [30]. Even assuming the convergence of the method, the numerical scheme produces for small value of the time step increment, numerical results which are not in accordance with the expected theoretical behavior. In particular, the stability of the equilibrium points and positivity of the solutions are not preserved. Previous research has shown that the process of transforming a classical model into a fractional one is very sensitive to the order of differentiation $\alpha$; for instance, a small change in $\alpha$ may result in a substantial change in the final result. From the numerical, it is clear that the approximate solutions depend continuously on the fractional derivative $\alpha$. In this paper, we introduced the same figures in [9] but we used Adams-Bashforth-Moulton algorithm. The approximate solutions $\mathrm{P}(\mathrm{t}), \mathrm{T}(\mathrm{t})$ and $\mathrm{Z}(\mathrm{t})$ are displayed in Figure 2 for the order of the fractional derivative $\alpha=0.95$. The results show that the concentrations of phytoplankton, toxic phytoplankton and zooplankton all reach their equilibrium values as time passes. An important feature of the fractional-order model is that it controls the speed at which the solution to equilibrium is reached. It follows from Theorem 3.2, that $\alpha^{*}=0.985237$. From Theorem 3.3, it is known that when $\alpha<\alpha^{*}$, the trajectories converge to the equilibrium point, as shown in Fig. 5-1, whereas when $\alpha$ is increased to exceed $\alpha^{*}$, the origin loses its stability, and a Hopf-type bifurcation occurs, as shown in Fig. 5-2. Furthermore, Fig. 6-2 illustrates that the system (2.1) has a stable cycle trajectory.
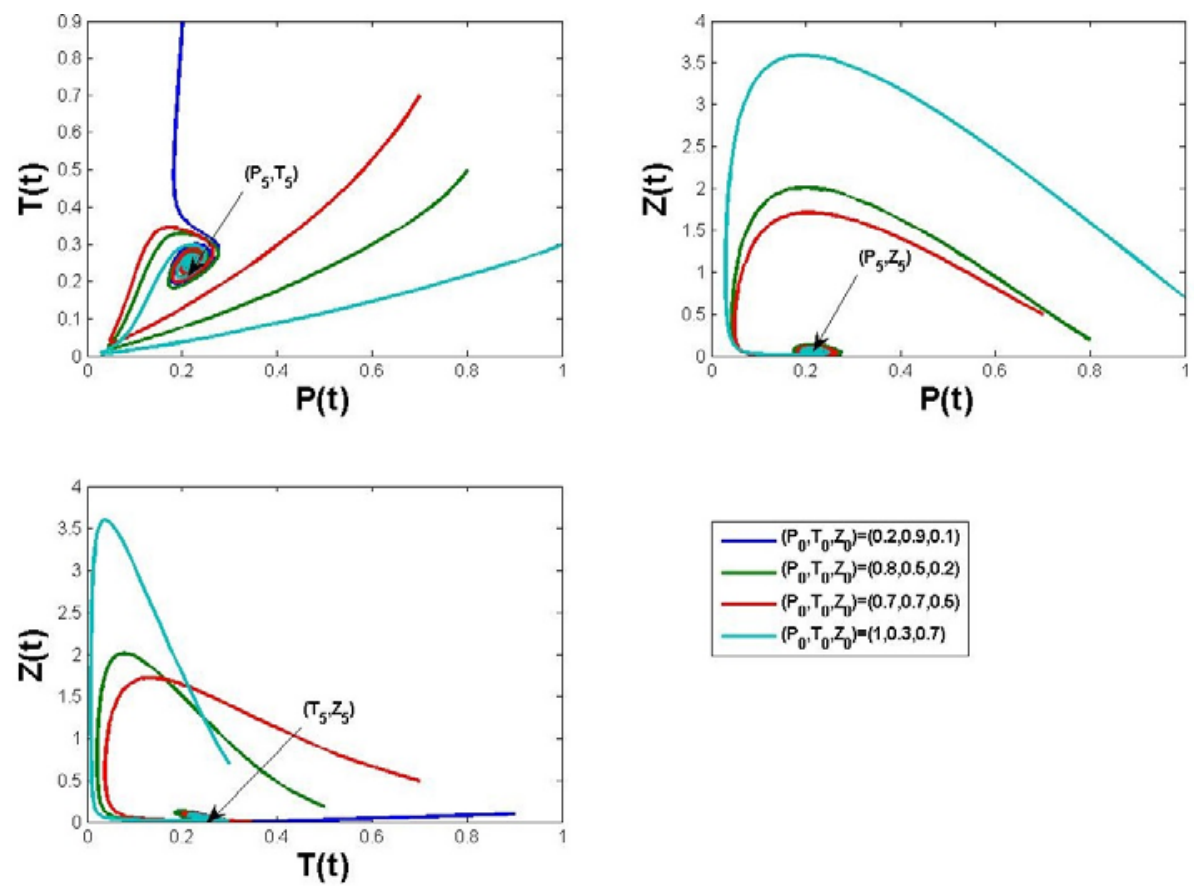

Figure 5-1. For various initial conditions and $\alpha=0.95$, the trajectory of system converges to the equilibrium point $\mathrm{E}_{5}$. 

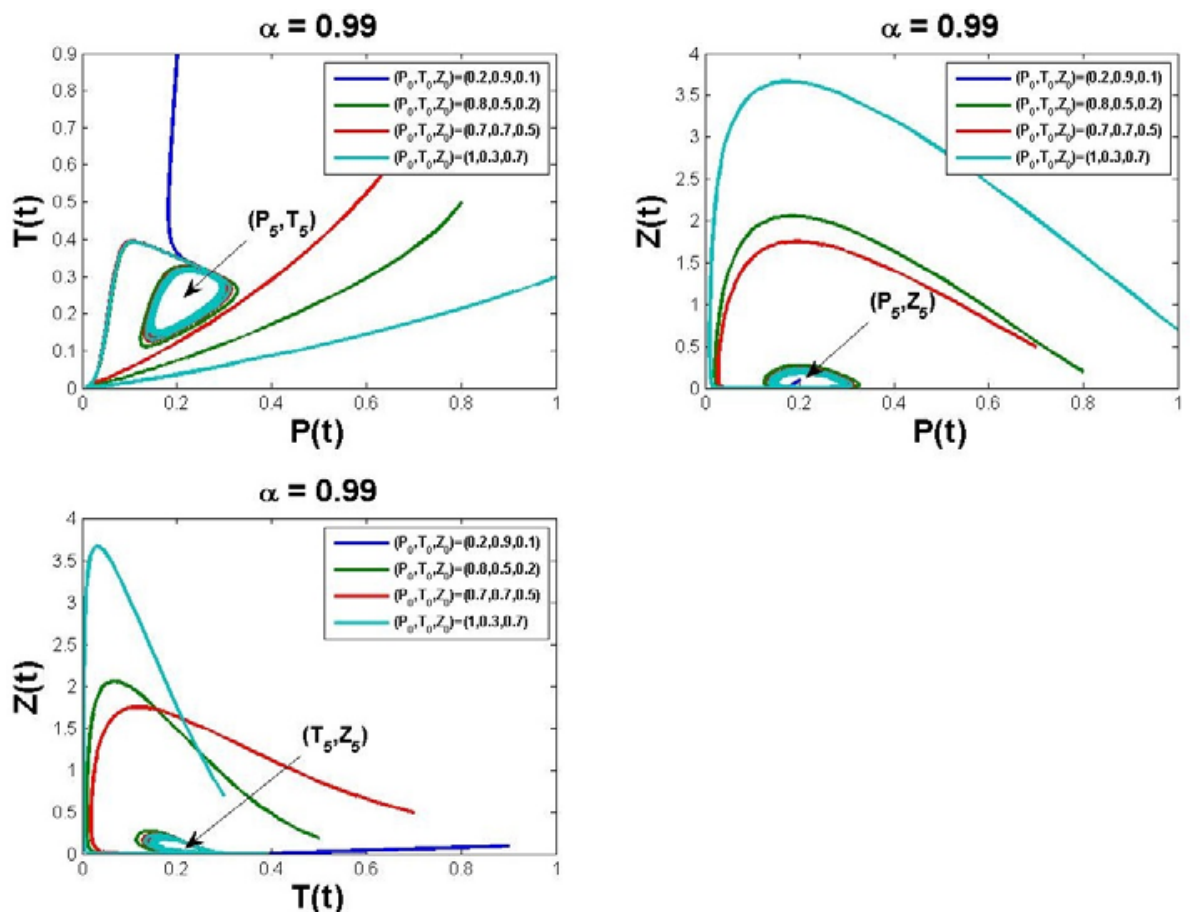

Figure 5-2. For various initial conditions and $\alpha=0.99$, the trajectory of system converges to an asymptotically stable limit cycle around $E_{5}$.
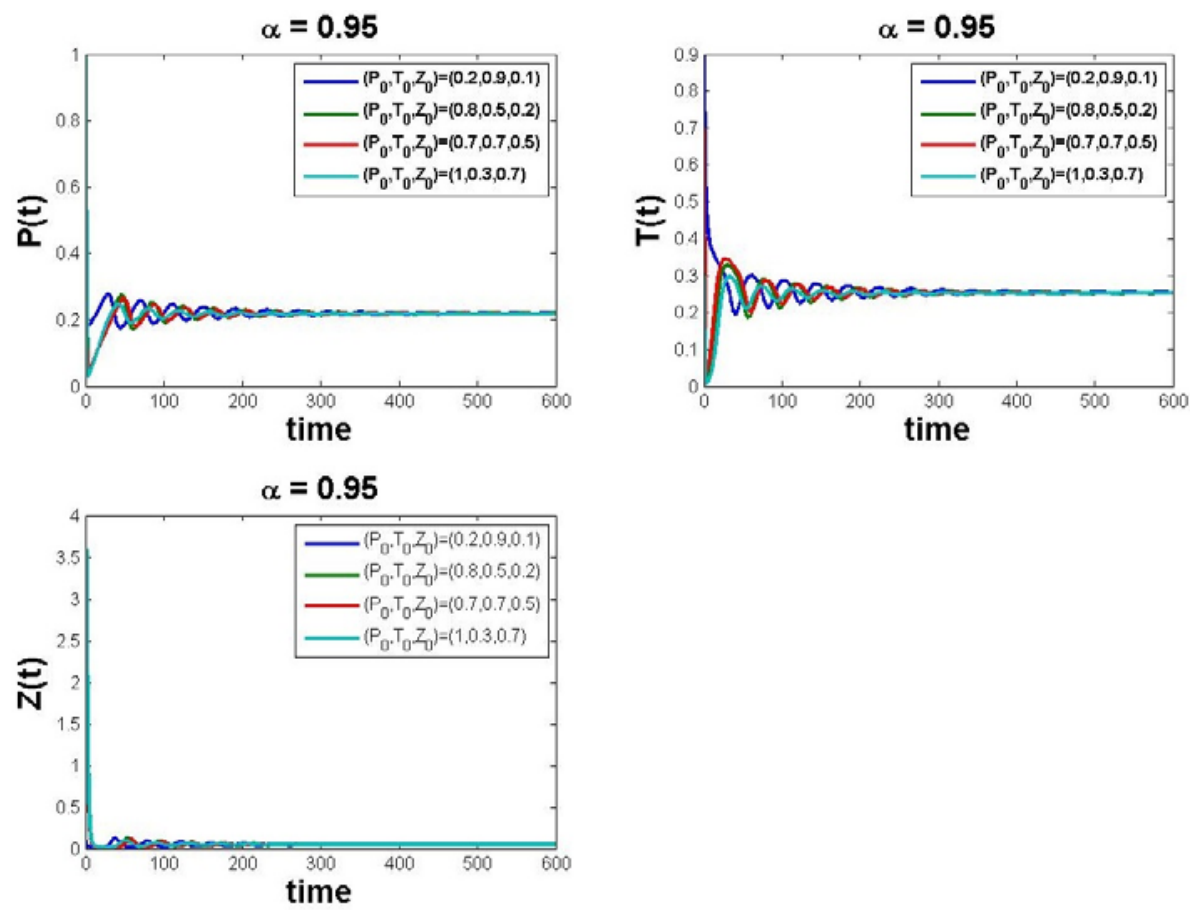

Figure 6-1. For various initial conditions and $\alpha=0.95$, the trajectory of system converges to the equilibrium point $E_{5}$. 

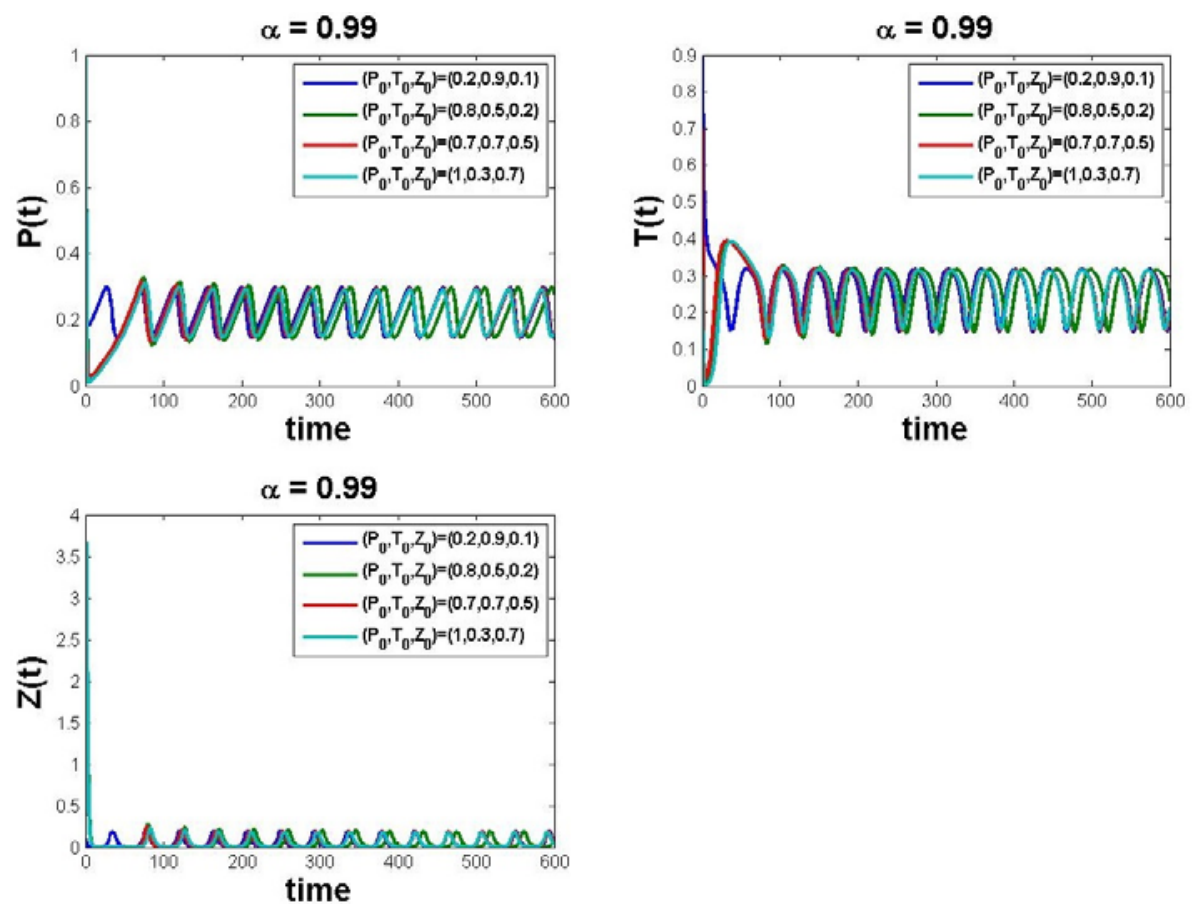

Figure 6-2. For various initial conditions and $\alpha=0.99$, the trajectory of system converges to an asymptotically stable limit cycle around $E_{5}$.
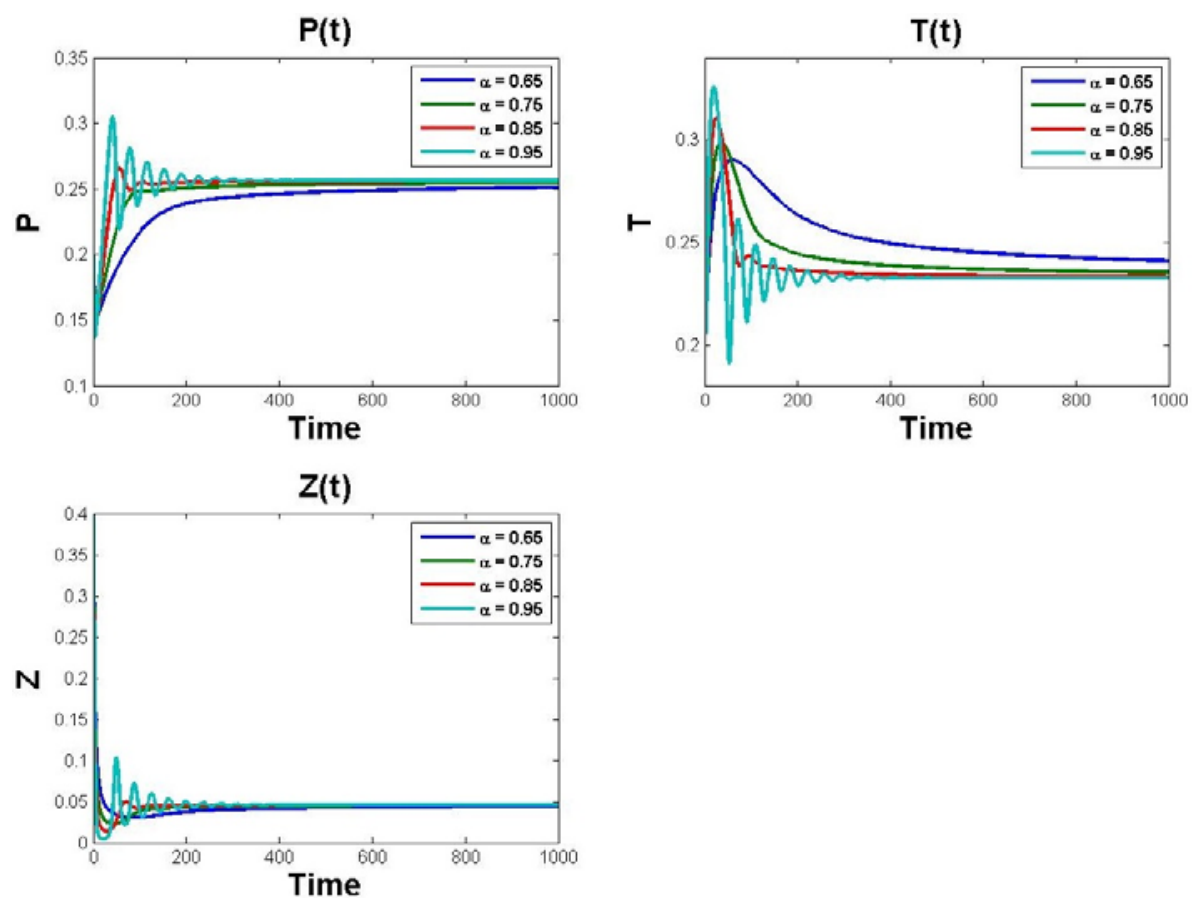

Figure 7. The trajectory of system depend continuously on the fractional derivative $\alpha$. 


\section{References}

1. http://www.arrancoast.com/research/aquaculture/51-what-are-toxic-phytoplankton-and-how-do-theyoccur.

2. https://en.wikipedia.org/wiki/Zooplankton.

3. E. Ahmed, A. M. A. El-Sayed, H. A. A. El-Saka, "Equilibrium points, stability and numerical solutions of fractional-order predator-prey and rabies models," J. Math. Anal. Appl, 325 (2007), 542-553.

4. M. S. Abd-Elouahab, N. E. Hamri, and J. Wang, "Chaos control of a fractional-order financial system," Mathematical Problems in Engineering, 2010 (2010), 18 pages.

5. H. Freedman, "Deterministic Mathematical models in Population ecology," Marcel Dekker, New York, 1980.

6. A. A. Kilbas, H. M. Srivastava, and J. J. Trujillo, "Theory and Applications of Fractional Differential Equations," Elsevier Science, Amsterdam, The Netherlands, 204 (2006).

7. I. Podlubny, "Fractional Differential Equations," Academic Press, New York, NY, USA (1999).

8. R. Garrappa, "Trapezoidal methods for fractional differential equations: Theoretical and computational aspects," Mathematics and Computers in Simulation, 110 (2015), 96-112.

9. M. Javidi, B. Ahmad, "Dynamic analysis of time fractional order phytoplankton-toxic phytoplankton zooplankton system," Ecological Modelling, 318 (2015), 8-18.

10. S. Kyu Choi, B. Kang, and N. Koo, "Stability for Caputo Fractional Differential Systems," Hindawi Publishing Corporation, Abstract and Applied Analysis, 2014 (2014), 6 pages.

11. D. Matignon, "Stability results for fractional differential equations with applications to control processing," Computational Engineering in Systems and Applications, Multi-conference, 2 (1996), 963-968.

12. E. Ahmed, A. M. A. El-Sayed, E. M. El-Mesiry and H. A. A. El-Saka, "Numerical solution for the fractional replicator equation," IJMPC, 16 (2005), 1-9.

13. E. Ahmed, A. M. A. El-Sayed, H. A. A. El-Saka, "On some Routh-Hurwitz conditions for fractional order differential equations and their applications in Lorenz," Rossler, Chua and Chen systems, Physics Letters A, 358 (2006), 1-4.

14. K. Diethelm, N. J. Ford, "Analysis of fractional differential equations," J Math Anal Appl, 256 (2002), 229-248.

15. K. Diethelm, N. J. Ford, A.D. Freed, "A predictor-corrector approach for the numerical solution of fractional differential equations," Nonlinear Dyn, 29 (2002), 3-22.

16. C. Li, C. Tao, "On the fractional Adams method," Computers and Mathematics with Applications, 58 (2009), 1573-1588.

17. R. S. Barbosa, J. A. T. MacHado, B. M. Vinagre, and A. J. Calderon, "Analysis of the van der Pol oscillator containing derivatives of fractional order," Journal of Vibration and Control, 13 (2007), 1291-1301.

18. D. Cafagna and G. Grassi, "Fractional-order Chua's circuit: time-domain analysis, bifurcation, chaotic behavior and test for chaos," International Journal of Bifurcation and Chaos, 18 (2008), 615-639.

19. A. E. Matouk, A. A. Elsadany, E. Ahmed, H. N. Agiza, "Dynamical behavior of fractional-order HastingsPowell food chain model and its discretization," Communications in Nonlinear Science and Numerical Simulation, 27 (2015), 153-167.

20. A. E. Matouk, A. A. Elsadany, "Dynamical behaviors of fractional-order Lotka-Volterra predator-prey model and its discretization," Journal of Applied Mathematics and Computing, 49 (2015), 269-283.

21. A. E. Matouk, A. A. Elsadany, "Dynamical analysis, stabilization and discretization of a chaotic fractionalorder GLV model," http://link.springer.com/article/10.1007/s11071-016-2781-6, 2015.

22. Vargas-De-León, "Volterra-type Lyapunov functions for fractional-order epidemic systems," Commun Nonlinear Sci Numer Simulat, 24 (2015), 75-85.

23. M. Elshahed and A. Alsaedi, "The Fractional SIRC model and Influenza A," Mathematical Problems in Engineering, Article ID 480378 (2011), 1-9.

24. M. Javidi, B. Ahmad, "A Study of a Fractional-Order Cholera Model," Appl. Math. Inf. Sci. 8,(2014) No. 5, 2195-2206.

25. M. Javidi, N. Nyamoradi, "Numerical Behavior of a Fractional Order HIV/AIDS Epidemic Model," World Journal of Modelling and Simulation, Vol. 9 (2013) No. 2, pp. 139-149.

26. M. Javidi, N Nyamoradi, "Dynamic analysis of a fractional order prey-predator interaction with harvesting," App. Math. Model. 37 (2013), 8946-8956. 
27. M. Javidi, N. Nyamoradi, "Numerical Chaotic Behavior of the Fractional Rikitake System," World Journal of Modelling and Simulation, Vol. 9 (2013), No. 2, pp. 120-129.

28. T.M. Atanackovic, B. Stankovic, "An expansion formula for fractional derivatives and its applications," Frac. Calculus Appl. Anal. 7 (3) (2004) 365-378.

29. T.M. Atanackovic, B. Stankovic, "On a numerical scheme for solving differential equations of fractional order," Mech. Res. Commun. 35 (7) (2008) 429-438.

30. J. Cresson, A. Szafrańska, "Discrete and continuous fractional persistence problems - the positivity property and applications," Communications in Nonlinear Science and Numerical Simulation, 44 (2017) 424-448. 